\title{
IoT Based Irrigation System using Natural Resource
}

\author{
Sushanth K J' ${ }^{1}$, Abdul Jabbar ${ }^{1}$, Mohammed Althaf ${ }^{2}$, Juraij Ahmed ${ }^{2}$, Thareef $^{2}$, Shabana Banu ${ }^{2}$ \\ Assistant Professor, Electronics and Communication Engineering Department, BIT, Mangalore, Karnataka, India ${ }^{1}$ \\ Students of Final Year, Electronics and Communication, Bearys Institute of Technology, Mangaluru, Karnataka, India ${ }^{2}$
}

\begin{abstract}
In nature, energy cannot be created or destroyed but its form can be changed. A sun tracking solar panel is used to harvest the natural resource into power storing device. The harvested power is used for smart irrigation, where the integrated soil moisture sensor senses the amount of water and depending upon the requirement it actuates the water pump. The fertilizer dispensing system dispenses the fertilizers on time basis. The irrigation field is protected from the intruders using PIR sensor. This system works in two modes automatic and manual mode, where in automatic mode the system works depending upon the sensor readings, whereas in manual mode a wireless interface is used between the user and the system. The system works depending upon the command given through wireless interface system in manual mode. The whole system is designed using ATMEGA2560.
\end{abstract}

Keywords: RFID, IR and PIR Sensors.

\section{INTRODUCTION}

In nature, energy cannot be created or destroyed but its form can be changed. A sun tracking solar panel is used to harvest the natural resource into power storing device. The harvested power is used for smart irrigation, where the integrated soil moisture sensor senses the amount of water and depending upon the requirement it actuates the water pump. The fertilizer dispensing system dispenses the fertilizers on time basis. The irrigation field is protected from the intruders using PIR sensor. This system works in two modes automatic and manual mode, where in automatic mode the system works depending upon the sensor readings, whereas in manual mode a wireless interface is used between the user and the system. The system works depending upon the command given through wireless interface system in manual mode. The whole system is designed using ATMEGA2560.

\section{METHODOLOGY AND PROPOSED SYSTEM}

In this proposed work IoT Based Irrigation System using solar as a natural resource. The power required to drive the entire integrated sensor networks and actuators are harvested from the sun tracking solar panel system continuously to a power storing device. Stored power is used to drive water pumps to pump water from bore well to a tank and the outlet valve of tank is automatically regulated using controller and moisture sensor to control the flow rate of water from the tank to the irrigation field which optimizes the use of water. Depending on the delay set in the microcontroller stored liquefied fertilizers are dispensed. And also, fencing applications suits remote areas provide an economical and practical solution to achieve maximum protection of field or particular areas. The above functions are monitored through Wi-Fi module integrated in the system in the smart devices.

\section{METHODOLOGY USED}

The Sun tracking solar panel consists of IR sensor, solar panel and DC geared motor. The IR sensor is fixed on the edge of the mountings. The DC geared motor connected to the panel rotates the panel in the direction of Sun. Panel is arranged in such a way that light on the IR sensor is compared and panel is rotated. The obtained power is stored to a battery.

\section{- IR Sensor Technology}

An IR sensor is a device which detects IR radiation falling on it. The principle of working of IR sensors is explained in the Fig. 1.

IR LED is used as IR transmitter and photodiode as receiver. When the vehicle passes through the booth, IR rays going to receiver are cut and signal is send to microcontroller [1]. 


\section{International Journal of Innovative Research in Electrical, Electronics, Instrumentation and Control Engineering \\ ISO 3297:2007 Certified}

Vol. 5, Issue 6, June 2017

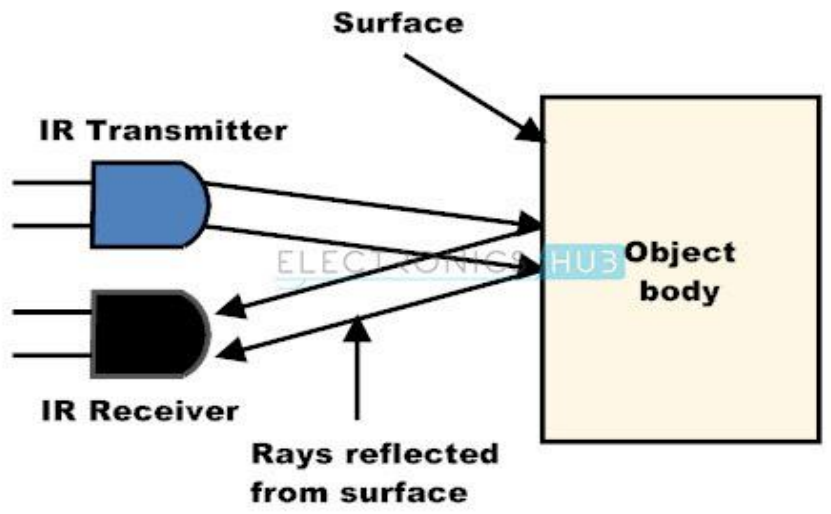

Fig.1: Block Diagram of IR Sensor

An IR sensor consists of an IR LED and an IR Photodiode; together they are called as Photo - Coupler or Opto Coupler. When the IR transmitter emits radiation, it reaches the object and some of the radiation reflects back to the IR receiver. Based on the intensity of the reception by the IR receiver, the output of the sensor is obtained.

\section{- PIR Sensor}

A passive infrared sensor (PIR sensor) is an electronic sensor that measures infrared (IR) light radiating from objects in its field of view. They are most often used in PIR-based motion detectors as shown in Fig 2.

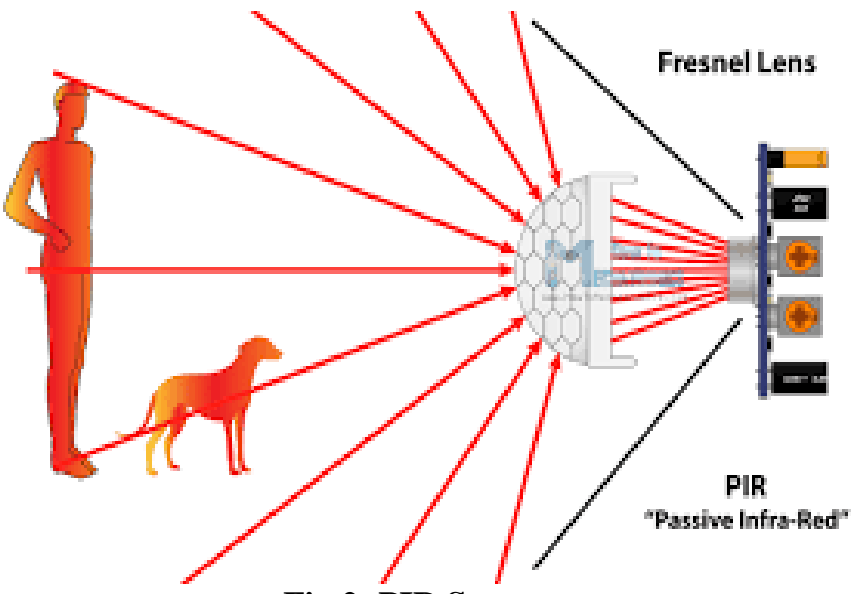

Fig.2: PIR Sensors

Pyroelectric devices, such as the PIR sensor, have elements made of a crystalline material that generates an electric charge when exposed to infrared radiation. The changes in the amount of infrared striking the element change the voltages generated, which are measured by an on-board amplifier. The device contains a special filter called a Fresnel lens, which focuses the infrared signals onto the element. As the ambient infrared signals change rapidly, the on-board amplifier trips the output to indicate motion.

\section{IMPLEMENTATION}

The implementation part of the project work includes the circuit schematic of entire system, the software used and the source code.

When irrigation mode is selected, the system allows to operate in two modes (shown in Fig:3) manual mode and automatic mode. In automatic mode, the system works with the sensor output. The soil moisture sensor senses soil condition. If the soil is in dry condition, the pump is on and if the soil is in wet condition, pump is off. Similarly, the PIR sensor checks for the intruders, if the intruders are present it turns on the buzzer and display the message as intruder detected. If no intruders are present it displays the message as no intruders detected. The fertilizer dispenser dispenses the fertilizer depending upon the delay time set. 
International Journal of Innovative Research in Electrical, Electronics, Instrumentation and Control Engineering

ISO 3297:2007 Certified

Vol. 5, Issue 6, June 2017

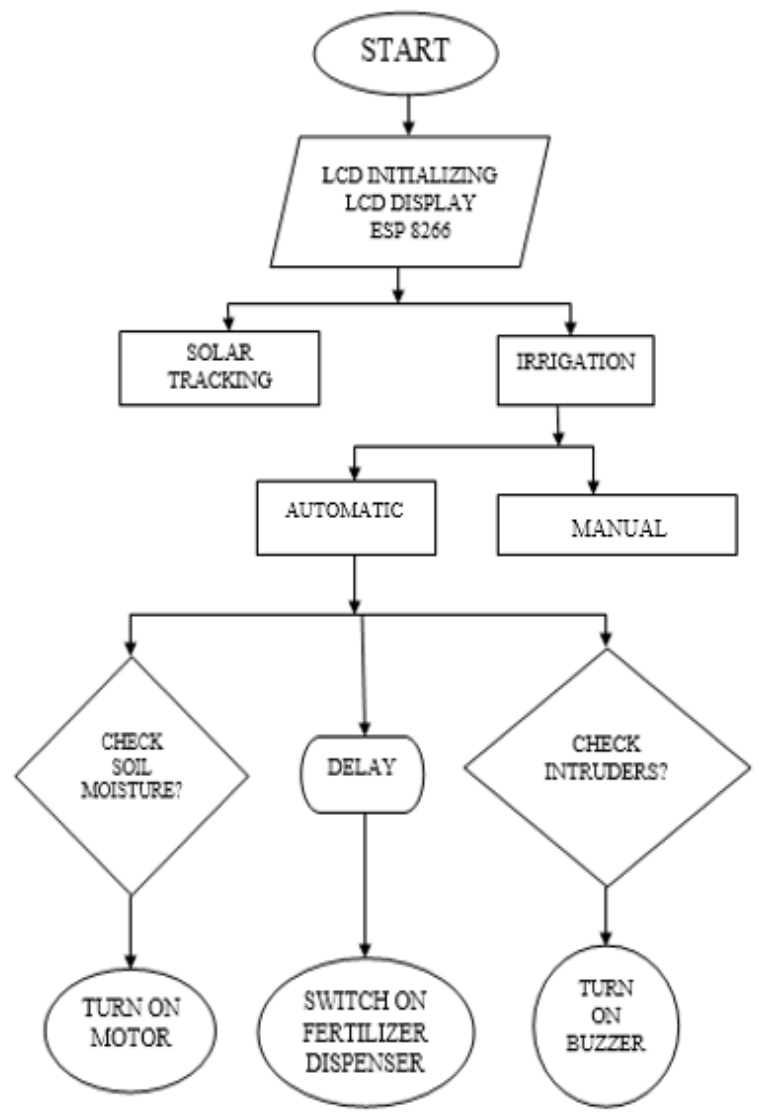

Fig.3 Flow Diagram of automatic Control of Irrigation System

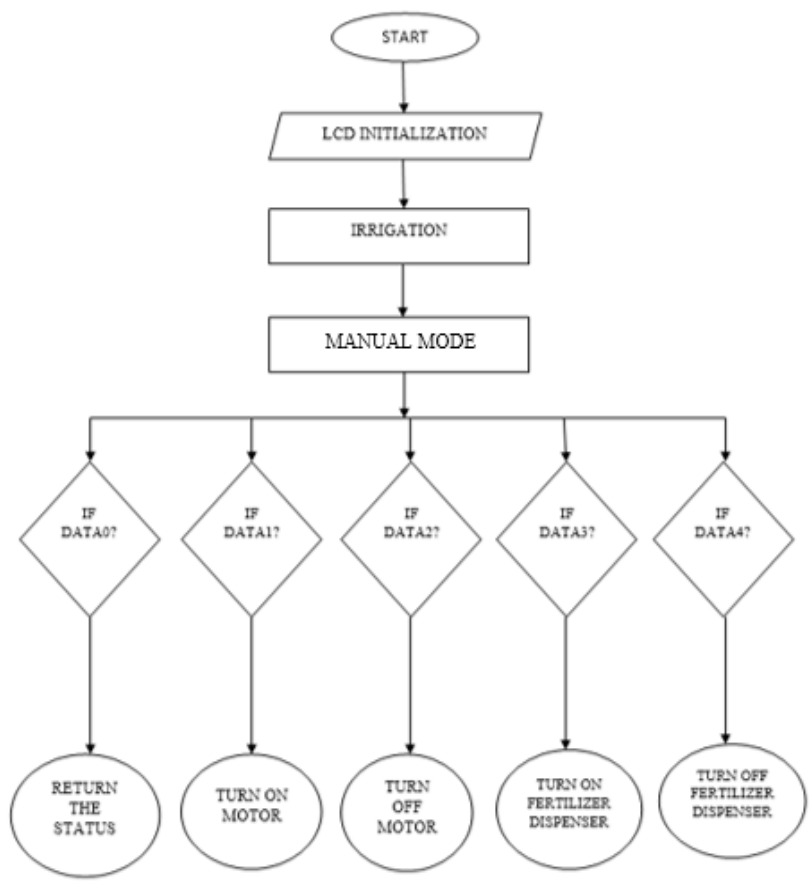

Fig. 4.Manual control system

In manual mode (shown in fig 4.4), the system acts as a server and connected to the user module where it can monitor the status and can be controlled by sending the control commands. 
IJIREEICE

\section{International Journal of Innovative Research in Electrical, Electronics, Instrumentation and Control Engineering \\ ISO 3297:2007 Certified \\ Vol. 5, Issue 6, June 2017}

\section{EXPERIMENTAL RESULTS}

The overall system module is shown in the Fig. 5 shows the intact module of power generation

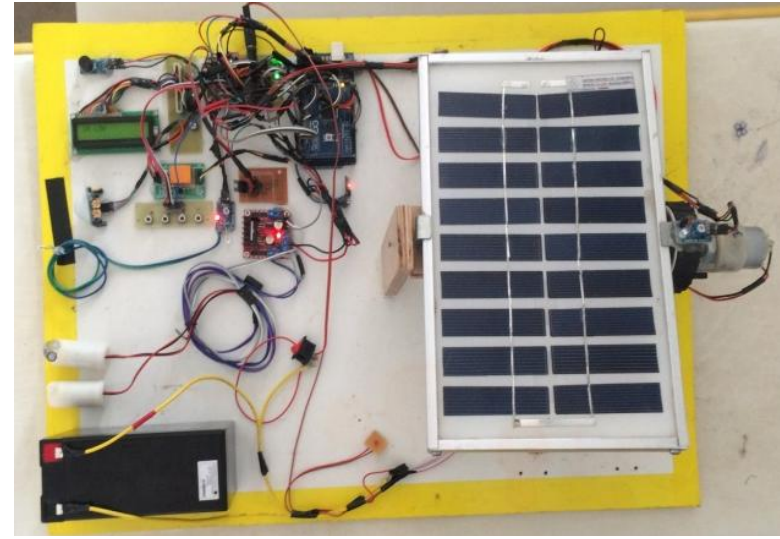

Fig.5. Overall view of proposed system

The overall system consists of a sun tracking solar panel. The solar panel is tilted by the DC geared motor depending on the IR sensor. The automatic irrigation system consists of soil moisture sensor, PIR sensor and water pumps. The system is monitored wirelessly using ESP8266.

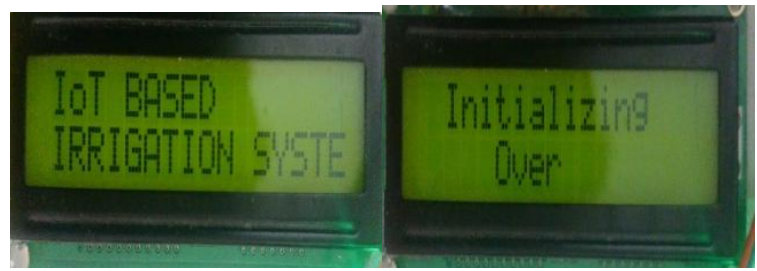

Fig 6. System Initialization

Above Fig 6. shows System is powered with $12 \mathrm{~V}$ battery. During powerup the system initializes the sensor network integrated to the system.

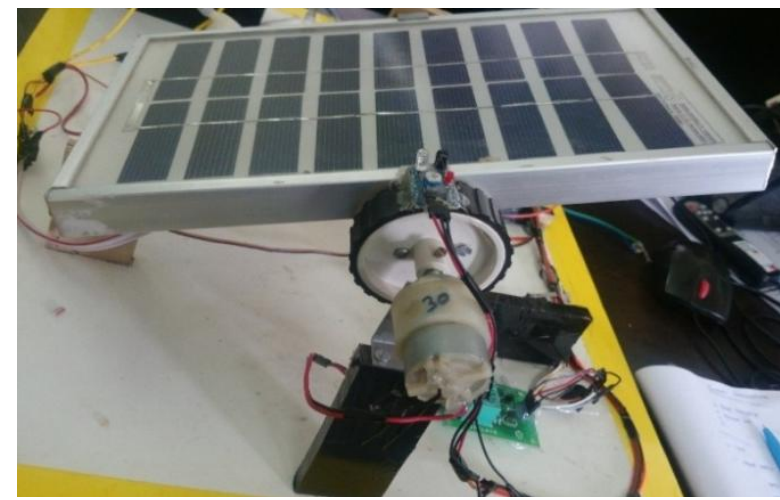

Fig 7. Sun tracking solar panel with IR sensor

The solar panel is mounted on a mechanical system which is driven by DC geared motor. The motor is actuated depending the IR sensor. IR sensor detects for the presence of IR rays as shown in Fig 7.

Esp8266 wi-fi module is interface for wireless communication. Manual control status shown in (fig 8).Wi-Fi is used for manual control of the system. Manual operation can be done by sending the following commands.

DATA0 -To Know the Status,

DATA1-Turn on The Pump

DATA2-Turn Off Pump

DATA3-Fertilizer On

DATA4-Fertilizer Off 


\section{IJIREEICE \\ International Journal of Innovative Research in Electrical, Electronics, Instrumentation and Control Engineering \\ ISO 3297:2007 Certified \\ Vol. 5, Issue 6, June 2017}

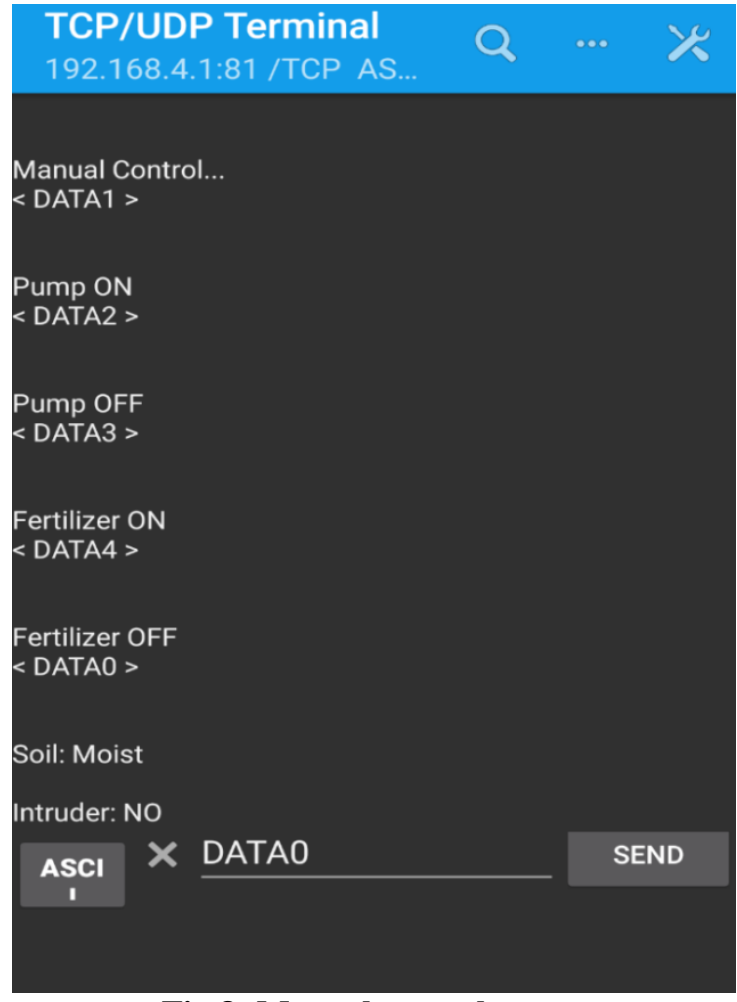

Fig 8: Manual control status

\section{CONCLUSION \& FUTURE SCOPE}

This project work will meet the challenges caused by the environmental changes. The system is designed to meet the requirements in the present irrigation system. The system uses freely available solar energy using sun tracking solar panel to harvest the power into power storing device. The power generated is used for irrigation system. Here irrigation system can be done in two modes- automatic mode and manual mode. Automatic mode helps to avoid unnecessary usage of water, since the water dispensing is depending on soil condition. For manual mode, the controlling is done through Wi-Fi interface. The system can work on by itself there is no need of external energy to derive the system.

In the future, the proposed system can be practically implemented for domestic use and some additional natural resources such as wind energy can also be added to it. By using this system, we can effectively utilize the natural resources. The excess generation of power can be sold.

\section{ACKNOWLEDGEMENT}

This work is carried out with the help of group of students and authors. We would like to thank all the faculty members, students and Prof. Mubeena Parveen Taj, HOD, Department of Electronics \& Communication, BIT, Mangaluru for their continuous Support.

\section{REFERENCES}

[1] R.Suresh, S.Gopinath, K.Govindaraju, T.Devika, N.SuthanthiraVanitha, "GSM based Automated Irrigation Control using Raingun Irrigation System”, International Journal of Advanced Research in Computer and Communication Engineering, Volume 3, Issue 2, February 2014.

[2] ChetanaA.Kestikar, RutujaM.Bhavsar," Automated Wireless Watering System”, International Journal of Applied Information Systems, Volume 2, Number 3, February 2012.

[3] S.Harishankar, R.Satish Kumar, Sudharsan K.P, U.Vignesh, T.Viveknath, "Solar Powered Smart Irrigation System", Advance in Electronic and Electrical Engineering, Volume 4, Number 4, 2014.

[4] BasavaSidramappaDhanne, Sachin Kedare, Shiva SidramappaDhanne, "Modern Solar Powered Irrigation System by Using ARM", International Journal of Reseach in Engineering and Technology, Volume 3, Issue 3, May 2014.

[5] Prof.RupaliS.Sawant, ShreejitGubre, Swathi Pillai, Monica Jain, "Solar Panel Based Automatic Plant Irrigation System", International Journal of Innovative Science, Engineering and Technology, Volume 2, Issue 3, March 2015. 